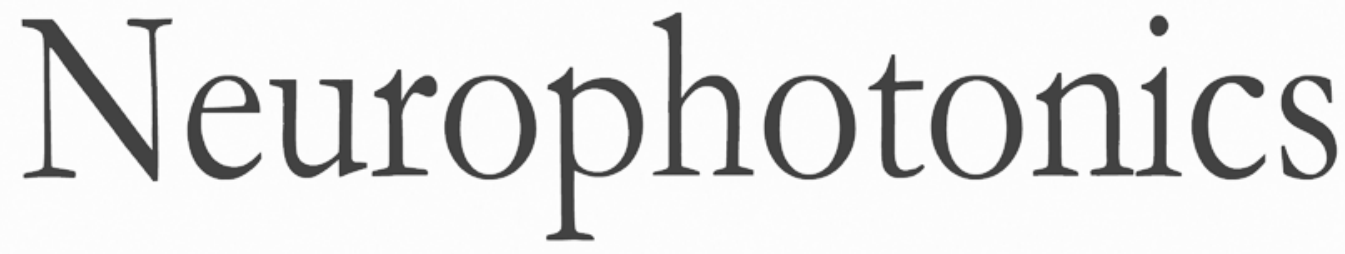

\title{
Restoring vision in mice with retinal degeneration using multicharacteristic opsin
}

Weldon Wright

Sivakumar Gajjeraman

Subrata Batabyal

Sanjay Pradhan

Sulagna Bhattacharya

Vasu Mahapatra

Ashutosh Tripathy

Samarendra Mohanty 


\title{
Restoring vision in mice with retinal degeneration using multicharacteristic opsin
}

\author{
Weldon Wright, Sivakumar Gajjeraman, Subrata Batabyal, Sanjay Pradhan, Sulagna Bhattacharya, \\ Vasu Mahapatra, Ashutosh Tripathy, and Samarendra Mohanty ${ }^{\star}$ \\ NanoScope Technologies LLC, Bedford, Texas, United States
}

\begin{abstract}
Retinal degenerative diseases, such as retinitis pigmentosa (RP) and dry age-related macular degeneration, have led to loss of vision in millions of individuals. Currently, no surgical or medical treatment is available, although optogenetic therapies are in clinical development. We demonstrate vision restoration using multicharacteristics opsin (MCO1) in animal models with degenerated retina. MCO1 is reliably delivered to specific retinal cells via intravitreal injection of adeno-associated virus (vMCO1), leading to significant improvement in visually guided behavior conducted using a radial arm water maze. The time to reach the platform and the number of error arms decreased significantly after delivery of MCO1. Notably, the improvement in visually guided behavior was observed even at light intensity levels orders of magnitude lower than that required for channelrhodopsin-2 opsin. Viability of vMCO1-treated retina is not compromised by chronic light exposure. Safe virus-mediated MCO1 delivery has potential for effective gene therapy of diverse retinal degenerations in patients. $\odot 2017$ Society of Photo-Optical Instrumentation Engineers (SPIE) [DOI: 10.1117/1.NPh.4.4.041505]
\end{abstract}

Keywords: vision restoration; optogenetics; retinitis pigmentosa; dry age-related macular degeneration.

Paper 17083SSPRR received Apr. 2, 2017; accepted for publication Jul. 24, 2017; published online Aug. 18, 2017; corrected Sep. 19, 2017.

\section{Introduction}

Loss of vision in millions of individuals ${ }^{1}$ is the result of the advanced stage of retinal dystrophies, such as dry age-related macular degeneration (dry-AMD) and retinitis pigmentosa (RP). There is currently no treatment available to cure retinal dystrophy or halt its progress. Since these retinal dystrophies are progressive and age-related disorders, the number of patients is expected to significantly increase in the near future. ${ }^{2}$ In these retinal disorders, the photoreceptors slowly degenerate and are unable to produce the signals that initiate visual perception. A number of gene therapy-based trials are underway, but it is still likely to be many years before this translates into possible treatment options. Retinal prostheses ${ }^{3-5}$ offer the possibility of restoring limited vision. ${ }^{6}$ Current systems, however, are limited by poor resolution, unstable long-term electrode and retinal tissue interface, retinal damage over a time period, and cellular overgrowth due to surgical implantation. Currently, high-resolution vision with large numbers of electrodes stimulating individual cells cannot be achieved due to high-power dissipation, which leads to heat production and leaves no chance for second implantation in case of failure (retinal damage).

Optogenetic sensitization ${ }^{7-12}$ of retinal cells ${ }^{13-15}$ has potential as an alternative to retinal implants. In addition to higher resolution, optogenetics has several advantages over electrical stimulation, such as cellular specificity (e.g., ON bipolar cells), and does not require intraocular surgery. However, clinical translation of optogenetic-enabled vision restoration suffers from the drawback of active stimulation by a light source having an intensity an order of magnitude higher than ambient light (i.e., bright ambient light level is $\sim 0.02 \mathrm{~mW} / \mathrm{mm}^{2}$ ) owing to

*Address all correspondence to: Samarendra Mohanty, E-mail: smohanty@ nanoscopetech.com the low photosensitivity of opsins deployed for the purpose. Such active stimulation has resolution limited by the resolution of the near-the-eye display and requires tracking of individual cells in the retina for achieving single-cell resolution. Further, chronic stimulation with high intensity blue light leads to retinal damage. Therefore, boosting up light intensity to high enough levels to activate conventional opsin-expressing cells, especially for chronic stimulation, may substantially damage the residual light-sensing function that might exist in the diseased or impaired retina of retinal dystrophy patients. The use of redshifted channelrhodopsin ${ }^{16-18}$ has promise for reducing the phototoxicity of retinal cells. Recently, to enhance sensitivity of cells toward ambient white light, a fusion vector (whiteopsin) containing three plasmids encoding opsins with spectrally separated activation peaks (ChR2 in blue, C1V1 in green, and ReaChR in red) $)^{17,18}$ was deployed. ${ }^{19}$ However, the use of multiple opsin constructs in same vector restricts its delivery to physical methods (e.g., optoporation ${ }^{20}$ or electroporation) since safe viral vectors [e.g., adeno-associated virus (AAV)] have limitations on carrying large size genes. Further, the light sensitivity still needs to be improved. ${ }^{21}$

To address this difficulty in optogenetic-enabled vision restoration, we have developed an ambient light activatable multicharacteristic opsin (MCO1) encoding gene, which is activated at the bright ambient light level. The MCO1 is packaged into a safe viral vector (AAV) with a fluorescent reporter (vMCO1-mCherry). The vMCO1 is intravitreally injected into the degenerated $r d 10$ mice retina, which is a well-established animal model of retinal dystrophy. Our results clearly demonstrate that the visually guided behavior that deteriorated with progressive retinal degeneration recovered back to a more nondegenerated level after vMCO1 treatment. More importantly,

2329-423X/2017/\$25.00 (C) 2017 SPIE 
this improved vision does not compromise the viability of vMCO1-treated retina.

\section{Material and Methods}

\subsection{Theoretical Modeling of the Three-Dimensional Arrangement of Amino Acid Chain}

We used a highly recognized web-based protocol, RaptorX, server for secondary structure and template-based tertiary structure prediction of our newly developed MCO1. The RaptorX uses a conditional neural field, a variant of conditional random fields, and a multiple template treating procedure to develop the following predicted structure of the MCO1 gene.

\subsection{Virus Carried Ambient Light Activatable Multicharacteristic Opsin (vMCO1) Synthesis}

The MCO1 gene was synthesized using a DNA synthesizer, and the sequence was verified. The synthesized plasmid was cloned into plasmid containing AAV terminal repeats multiple cloning site vector via its BamH1 and Sall sites. AAV physical titers were obtained by quantitative polymerase chain reaction using primers designed to selectively bind AAV inverted terminal repeats. TCID50 assay was conducted according to American Type Culture Collection protocol. Verification of the purity of the purified virus was confirmed by sodium dodecyl sulfate/polyacrylamide gel electrophoresis.

\subsection{Cell Culture and MCO1 Plasmid Transfection}

HEK293 cells were transfected with MCO1 plasmids using Lipofectamine 3000 (Invitrogen). After transfection, HEK293 cells were cultured in Petri dishes and maintained in Dulbecco's modified Eagle's medium/F-12 with $10 \%$ fetal bovine serum, $0.2 \mathrm{mg} / \mathrm{mL}$ streptomycin, and $200 \mathrm{U} / \mathrm{mL}$ penicillin. The cultures were maintained at $37^{\circ} \mathrm{C}$ in a $5 \% \mathrm{CO}_{2}$ humidified atmosphere. Visualization of the reporter (mCherry) fluorescence was carried out under epifluorescence (Nikon Eclipse TS 100) or confocal microscopy (Olympus Fluoview FV1000).

\subsection{Optogenetic Stimulation}

A single-mode optical fiber coupled to a supercontinuum laser source (SuperK Compact, NKT Photonics) delivered the light to the sample via fiber optic cable (SuperK FD-2) that transmits light in the visible spectrum for in-vitro optogenetic stimulation. The light intensity was varied by a current controller. A power meter (Newport) was used to quantify the light intensity at the sample plane. The light pulse width was controlled by a shutter, synchronized with the electrophysiology recording system (Molecular Devices). Cells, transfected with MCO1, were incubated with all-trans retinal $(1 \mu \mathrm{M})$ for $6 \mathrm{~h}$ before conducting the patch-clamp experiments.

\subsection{Patch-Clamp Recording Setup}

Inward photocurrents in MCO1-transfected cells were recorded using the patch clamp. The patch-clamp recording setup consists of an inverted Nikon fluorescence microscope platform and an amplifier system (Axon Multiclamp 700B, Molecular Devices). Micropipettes were pulled using a two-stage pipette puller (PC10 , Narshige) to attain resistance of 3 to $5 \mathrm{M} \Omega$ when filled with intracellular solution. The micropipette electrode was mounted on a micromanipulator. The micropipette was filled with a solution containing (in $\mathrm{mM}$ ) $130 \mathrm{~K}$-gluconate, $7 \mathrm{KCl}, 2 \mathrm{NaCl}$, $1 \mathrm{MgCl}_{2}, 0.4$ EGTA, 10 HEPES, 2 ATP-Mg, 0.3 GTP-Tris, and 20 sucrose. The micropipette electrode was mounted on a micromanipulator to patch onto cells. The extracellular solution contained (in $\mathrm{mM}$ ) $150 \mathrm{NaCl}, 10$ glucose, $5 \mathrm{KCl}, 2 \mathrm{CaCl}_{2}$, and $1 \mathrm{MgCl}_{2}$ was buffered with $10 \mathrm{mM}$ HEPES (pH 7.3). Photocurrents were measured while holding cells in a voltage clamp at $-70 \mathrm{mV}$. The electophysiological signals from the amplifier were digitized using Digidata 1440 (Molecular Devices), interfaced with patch-clamp software (pClamp, Molecular Devices). The light pulse width was synchronized with the electrophysiology recording system, controlled by Axon Instruments Digidata system. pClamp 10 software was used for data analysis.

\section{6 rd10 Mice Preparation}

The advanced stage of retinal dystrophy is characterized by degeneration of photoreceptors in the eye that hinders visual ability by nonfunctional neuronal activation and transmission of signals to the visual cortex. The $r d 10$ mice (retinal degeneration 10, spontaneous missense point mutation in Pde6b) have a later onset and progressive retinal degeneration as compared with $r d l$ mice. This is important for sight restoration purposes because it gives a window of time during which the visual cortex can learn how to interpret visual signals. $r d 10$ mice and C57BL/ $6 \mathrm{~J}$ wild-type mice (control) were obtained from Jackson laboratory and bred in the animal facilities at the Nanoscope Technologies. Mice were maintained on a 12:12 light cycle.

Nanoscope Technologies's Institutional Animal Care and Use Committee approved the experimental protocol involving animals. The methods were carried out in accordance with the relevant guidelines.

\subsection{Intravitreal Injection of vMCO1 to rd10 Mouse Retina}

An aseptic technique was used for all surgical procedures, and surgical tools were sterilized with autoclave. The $r d 10$ mouse $(n=10)$ was anesthetized with isoflurane $(2 \%$ to $3 \%)$ and mounted on a stereotaxic frame. One drop each of $1 \%$ atropine sulphate, $1 \%$ Mydriacyl ophthalmic solution, and $10 \%$ phenylephrine hydrochloride were instilled into the eye of the animal. The vMCO1 $(1 \mu \mathrm{L})$ solution was injected by a sterilized needle of a Hamilton microsyringe inserted through the sclera into the vitreous cavity (intravitreal injection). The vMCO1 solution was injected into both sides of the eye. The cornea was kept moist with a balanced salt solution during the entire surgical procedure.

\subsection{Viability of Retinal Cells Subsequent to Chronic Light Exposure}

Long-term (4 weeks) viability of retinal cells in the transfected retina was evaluated after chronic light stimulation $(8 \mathrm{~h} /$ day $)$. The mice were placed inside a chamber containing white light sources, providing intensity of $0.1 \mathrm{~mW} / \mathrm{mm}^{2}$ on the floor of the chamber. Four weeks after light exposure, the transfected $(n=5$ $r d 10$ mice) as well as wild-type (control, $n=5)$ mice were euthanized, and the eye tissue was harvested for retina extraction. The explanted retina was immunostained using an apoptotic marker (cleaved caspase-3)/4',6-diamidino-2-phenylindole 
(DAPI) and imaged using confocal microscopy (Olympus Fluoview FV1000). The number of total cells versus apoptotic retina cells was compared between transfected and control groups.

\subsection{Water Maze Behavioral Assessment}

$r d 10$ mice $(n=5)$ with retinal degeneration were used inclusion criteria of age $>8$ weeks. For type I error rate $\alpha=0.05$ and $80 \%$ to $90 \%$ power, the required number of animals is 3 to 4 /group. After vMCO1 injection, mice were tested with a radial arm water maze to determine behavioral restoration of vision in rd10 mice with vMCO1-sensitized retinal cells. Briefly, mice are placed into the center of the maze, and a platform is placed just below the water's surface at the end of one of the arms. The mice rapidly learn to determine the location of the platform by utilizing visual cues [light-emitting diodes (LEDs) emitting light with visible spectrum]. The platform (in one of the arms) provided a reward to them where they can rest instead of having to swim. The time to reach the platform and the number of error(s) made before finding the platform were quantified for both light $\mathrm{ON}$ and OFF conditions. Data (video) recording was stopped once the mice found the platform or before $60 \mathrm{~s}$ of dropping the mice in water to prevent the mice from getting tired of swimming. For each mouse, $n=5$ trials were conducted in each location (i.e., center, side arm, and edge of the water maze). The selection of dropping site (center, side, and edge) was random for each mice and each trial. The exclusion criterion consists of a mouse that does not swim (and floats).

\section{Results and Discussion}

\subsection{Ambient Light Activatable Multicharacteristic Opsin}

The present approaches for optogenetic restoration of vision have used ChR $2,{ }^{13}$ which is not activatable at the ambient light level and, therefore, not able to generate action potential (AP) in an ambient environment. Thus, all attempts at restoring vision by optogenetic stimulation so far has focused on the development and implementation of an active array of intense light sources (e.g., LED array ${ }^{22}$ ) to activate opsin-sensitized cells in the retina. However, considerable concerns about viability of opsin-sensitized neurons as well as residual photosensitive cells under extended exposure to such intense light exist. We have developed an ambient light activatable MCO1. Figure 1(a) shows the domain architecture of MCO1 with reporter (mCherry). In Fig. 1(b), we show a typical circular map showing the insertion of the MCO1 gene cloned at the restriction sites (BamH I and Sal I). Figure 1(c) shows the three-dimensional (3-D) arrangement of amino acid chains of MCO1 obtained by theoretical modeling using RaptorX. Figure 1(d) shows the agarose gel electrophoresis of the mGluR6-MCO1-mCherry gene (2915 bp) after restriction enzyme digestion with BamH1 and Sal1. Multicharacteristics opsin (MCO1) has high photosensitivity with unique spectral and temporal characteristics to generate significant current in response to ambient light (Fig. 2). (a)

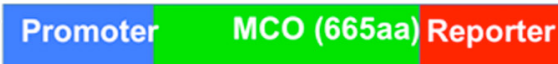

(b)

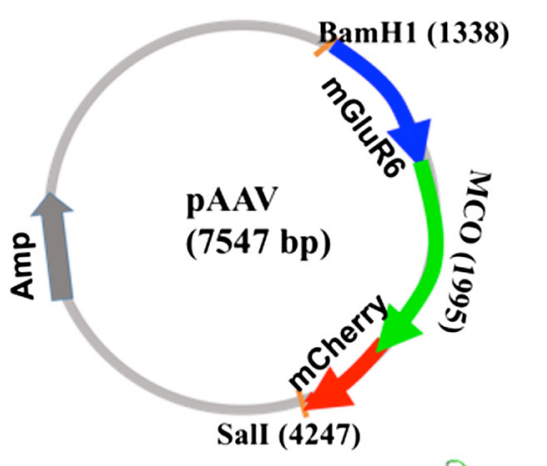

(c)

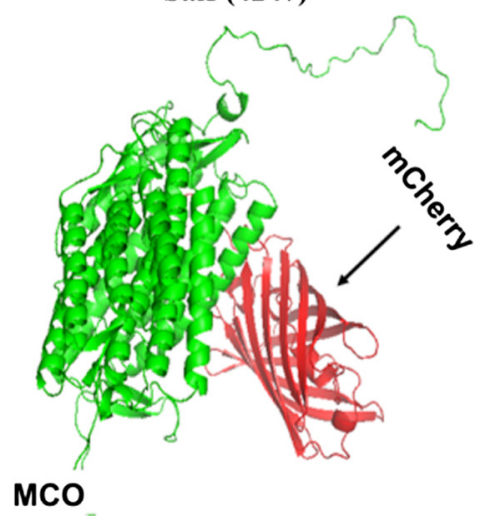

(d)

$5 \mathbf{k b}$

$3 \mathbf{k b}$

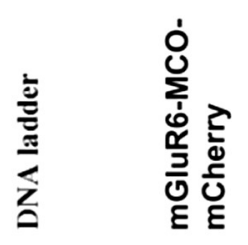

$1 \mathrm{~kb}$

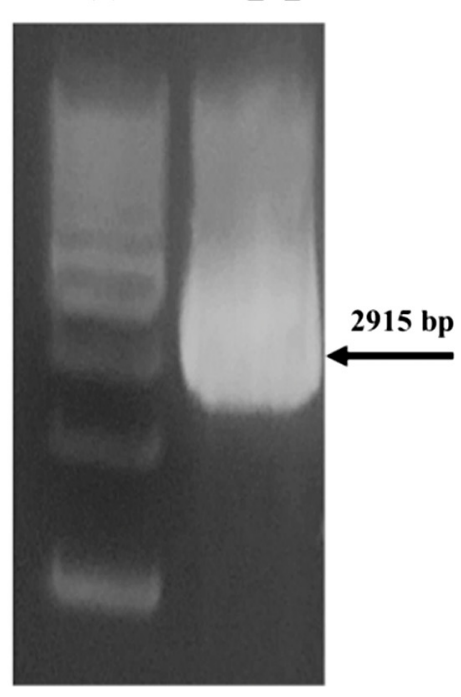

Fig. 1 Ambient light activatable MCO1: (a) domain architecture of MCO1 with reporter protein, (b) a typical circular map showing the insertion of MCO1 gene cloned at the restriction sites (BamH I and Sal I), (c) theoretical modeling of the 3-D arrangement of amino acid chains of MCO1 using webbased protocol (RaptorX), and (d) agarose gel electrophoresis of mGluR6-MCO-mCherry gene (2915 bp) after restriction enzyme digestion with BamH1 and Sal1. 
(a)

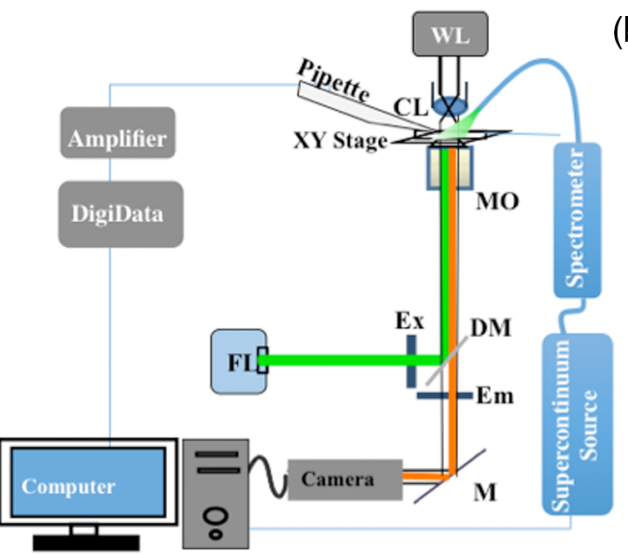

(c) 6000

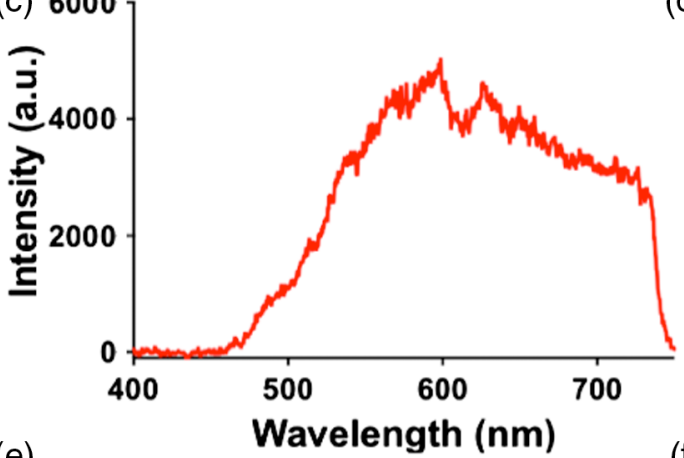

(e)

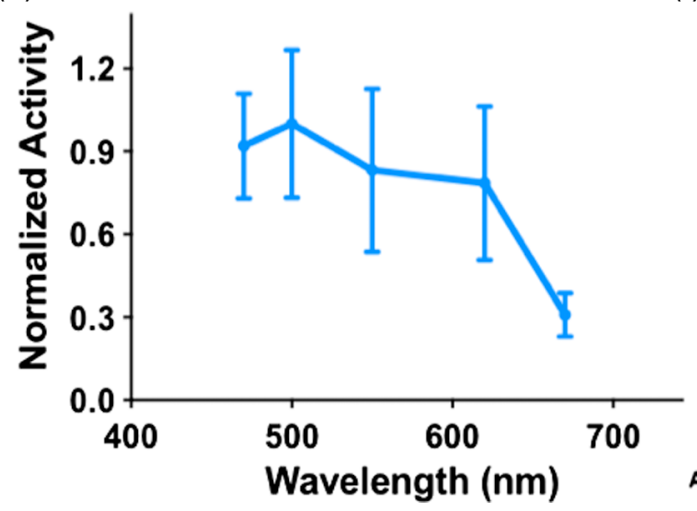

(b)

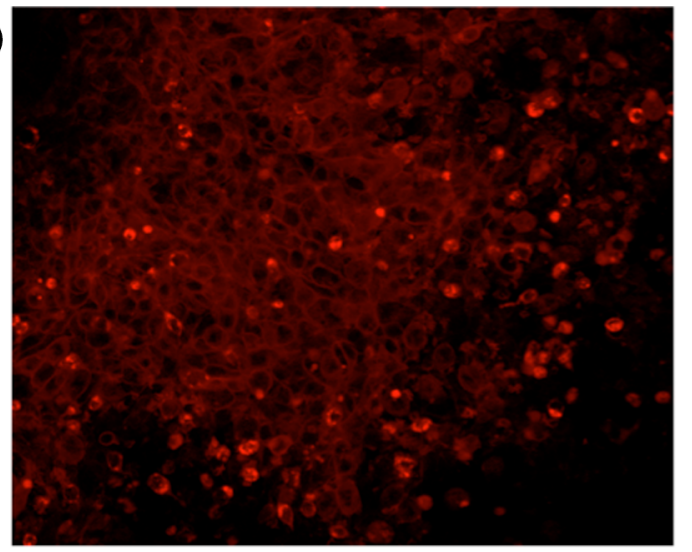

(d)

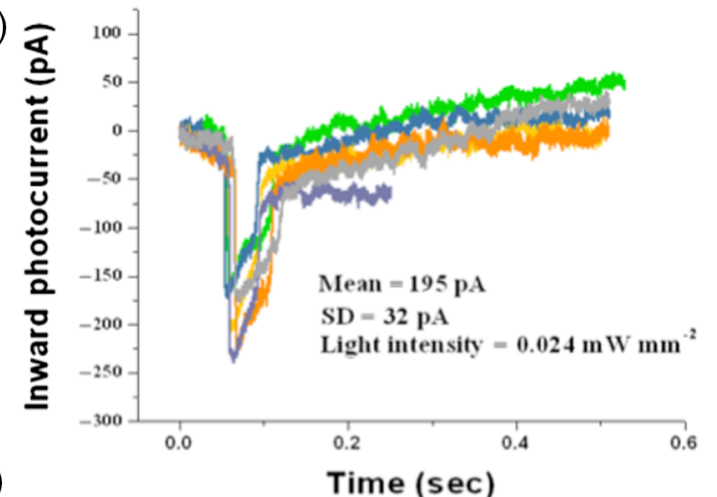

(f)

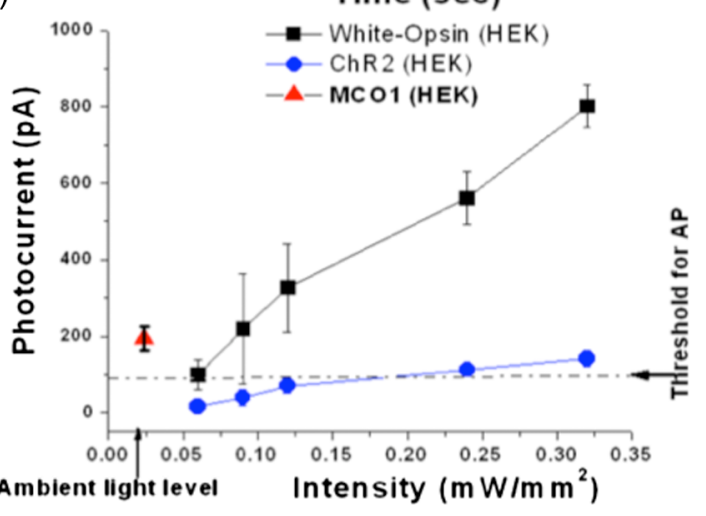

Fig. 2 Expression and functioning of MCO1: (a) experimental setup for opto-electrophysiology of MCO1transfected cells. CL, condenser; MO, microscope objective; FL, fluorescence excitation source; EX, excitation filter; DM, dichroic mirror; EM, emission filter; and M, mirror. (b) Fluorescence upon lipofection of MCO1-mCherry into HEK293 cells, (c) spectrum of light used for stimulation of MCO1-expressing cells, (d) inward current profiles in MCO1-expressing cells in response to light (average intensity: $0.024 \mathrm{~mW} / \mathrm{mm}^{2}$ ) measured by patch-clamp electrophysiology, (e) activation spectrum of MCO1 (average $\pm \mathrm{SEM}$ ), and (f) comparison of MCO1 photocurrent with ChR2 and white-opsin. Inward photocurrent in MCO-sensitized cells at ambient light level is above the threshold for AP. For white-opsin, $n=$ 6 cells, for ChR2, $n=6$ cells, and for MCO1, $n=3$ cells (average \pm SD).

\subsection{Sensitivity of Multicharacteristic Opsin to Light}

To determine the light-dependent inward photocurrent, the MCO1-expressing cells were exposed to pulses of light with various intensities ranging from 0.01 to $0.03 \mathrm{~mW} / \mathrm{mm}^{2}$. Figure 2(a) shows the schematic of the experimental setup for optical stimulation and electrical recording. Fluorescence image of HEK293 cells after $48 \mathrm{~h}$ of lipofection of MCO1-mCherry plasmids is shown in Fig. 2(b). Figure 2(c) shows the spectrum of the light used for stimulation of MCO1-expressing cells. Representative inward photocurrent profiles in HEK cells transfected with MCO1 are shown in Fig. 2(d). To obtain the activation spectrum of MCO1, the inward photocurrent was measured using stimulation light at different wavelengths (with bandwidth: $30 \mathrm{~nm}$ ). In Fig. 2(e), we show the normalized activation spectrum of MCO1. The inward photocurrent was found to be an order of magnitude higher in MCO1-sensitized cells than that in the $\mathrm{ChR} 2$ expressing cells. Figure 2(f) shows a comparison of MCO1 photocurrent with ChR2 and white-opsin. ${ }^{21}$ The inward photocurrent $(195 \pm 32 \mathrm{pA})$ in MCO1-sensitized cells at an ambient light level $\left(0.02 \mathrm{~mW} / \mathrm{mm}^{2}\right)$ is above the threshold for $\mathrm{AP}$ unlike that in cells sensitized with ChR2 and white-opsin. ${ }^{21}$

It may be noted that for a good fidelity of the light-evoked spiking of opsin-sensitized cells, faster response time is 
required. The $\mathrm{ON}$ response time of an ambient light activatable MCO1 [Fig. 2(d)] is measured to be $2.94 \pm 0.70 \mathrm{~ms}$, which is similar to that measured for other fast opsins. ${ }^{23}$ However, the ON response time depends on the intensity of activation light and is known to increase as the light intensity decreases. ${ }^{18}$

\subsection{Transfection Using Viral Vector Carried Multicharacteristic Opsin (vMCO1)}

First, delivery of MCO1 by AAV was confirmed in-vitro in HEK cells [Fig. 3(a)]. In-vivo viral transfection was conducted for delivery of the MCO1 to the bipolar cells in the retina of the rd10 mouse model. We performed intravitreal injection of $1 \mu \mathrm{L}$ solution of vMCO1 (in both eyes), in which the MCO1 was under control of a specific promoter (mGluR6), targeted for ON retinal bipolar cells. Uniformity of MCO1 expression was confirmed by the 3-D reconstruction from the confocal mCherry expression in $z$-slices of the whole retinal cup in rd10 mice injected with vMCO1 intravitreally [Fig. 3(b)].

\subsection{Effect of Improved Light Sensitivity of the Retina on rd10 Mice Behavior in the Visual Water Maze}

For testing the spatial memory and learning capabilities of vMCO1-treated rd10 mice toward light, a visual radial arm water maze was used. ${ }^{24}$ To determine behavioral restoration of vision in vMCO1-treated $r d 10$ mice, one week before injection of vMCO1, mice were tested with light ON and OFF conditions in a radial visual water maze. The light intensity used in these behavioral studies was $7 \mu \mathrm{W} / \mathrm{mm}^{2}$ at the center and $<5 \mu \mathrm{W} / \mathrm{mm}^{2}$ in the edge/side arms of the radial water maze. This corresponds to retinal illumination below $0.01 \mu \mathrm{W} / \mathrm{mm}^{2}{ }^{25}$ Figure 4(a) shows a time-lapse imaging of a $r d 10$ mouse navigating the visual water maze with white LED light ON before intravitreal vMCO1 injection. The mice were again assayed with both light $\mathrm{ON}$ and $\mathrm{OFF}$ conditions after intravitreal injection of vMCO1. Figure 4(b) shows time-lapse imaging of the same rd10 mouse navigating the visual water maze six weeks after vMCO1 injection at same LED light intensity. The time traveled by the vMCO1-transfected mice before arriving at the platform was much shorter $[<20 \mathrm{~s}$, Fig. 4(b)] than before injection [>60 s, Fig. 4(a)].

Consistent with the number of error arms, the mean number of arms swam by the vMCO1-transfected mice before they reached the platform is significantly smaller $(<1)$ than that of the mice before transfection $(>2)$. Figures 4(c)-4(e) show the change in latency to find the platform by the vMCO1-transfected $\mathrm{rd10}$ mouse, with and without light, dropped at the center (c), side arms (d), and edge (e). Notably, the improvement in visually guided behavior was observed at a light intensity level of $\sim 10 \mu \mathrm{W} / \mathrm{mm}^{2}$ (retinal illumination level $<0.01 \mu \mathrm{W} / \mathrm{mm}^{2}$ ) an order of magnitude lower than that required for mice sensitized with ChR2 opsin. Figures 4(f)-4(h) show the number of error arms traversed by the mice dropped at different locations before finding the platform in the presence and absence of light: center (f), side arms (g), and edge (h). The data collected from one mouse that was found to be floating most of the time, instead of swimming, were not included.

\subsection{Chronic Light Exposure Did Not Compromise Viability of vMCO1-Treated Retina}

Chronic exposure of MCO1-transfected retina to light may raise concern about their viability. We therefore exposed vMCO1treated $r d 10$ mice to an order of magnitude higher intensity white light (i.e., $0.1 \mathrm{~mW} / \mathrm{mm}^{2}$ ) than that of the targeted ambient light level (i.e., $0.01 \mathrm{~mW} / \mathrm{mm}^{2}$ ). Long-term (4 weeks) viability of retinal cells in the transfected retina was evaluated after chronic light stimulation ( $8 \mathrm{~h} /$ day). It may be noted that, similar to the wild-type mice, the vMCO1-treated $r d 10$ mice avoided bright light by blocking the light via the creation of a hill out of the bedding material [as shown by arrow, Fig. 5(a)].

Four weeks after light exposure, the transfected $r d 10$ mice as well as the wild-type mice were euthanized, and the eye tissue was harvested for retina extraction. The retinal cups were immunostained using an apoptotic marker (caspase-3) and imaged using confocal microscopy. Figure 5(b) shows the caspase-3 (green)- and DAPI (blue)-stained retina of vMCO1-treated rd10 mice after chronic light exposure. Figure 5(c) shows the
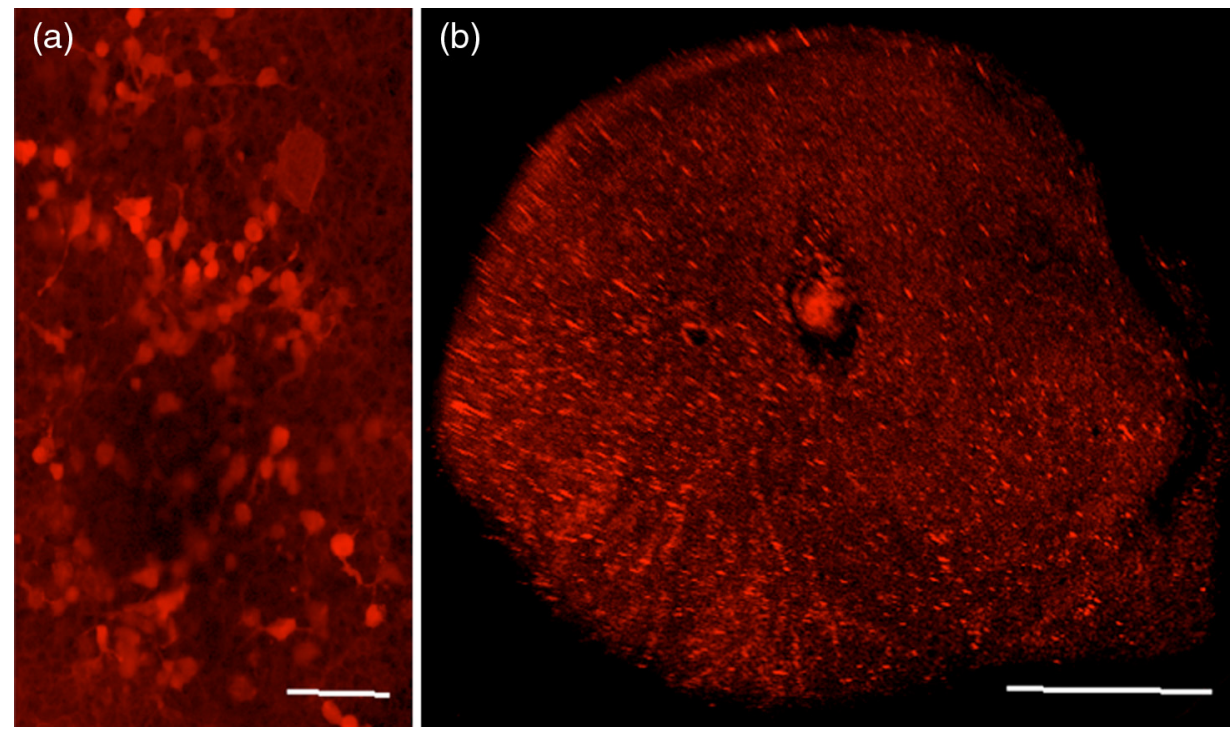

Fig. 3 Three-dimensional reconstruction of vMCO1-mCherry expression in cells and retina: (a) HEK293 cells, scale bar: $50 \mu \mathrm{m}$ and (b) whole retinal cup, scale bar: $0.8 \mathrm{~mm}$. 
(a)

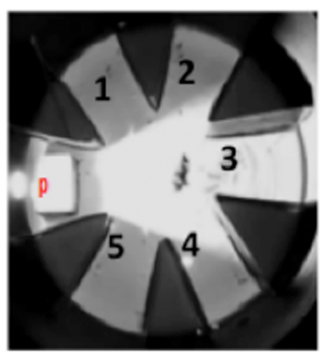

Os

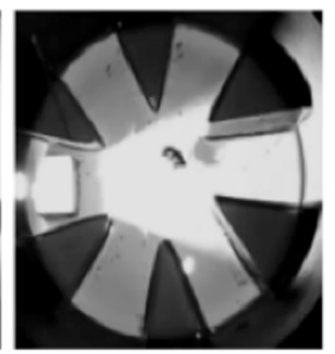

$15 \mathrm{~s}$

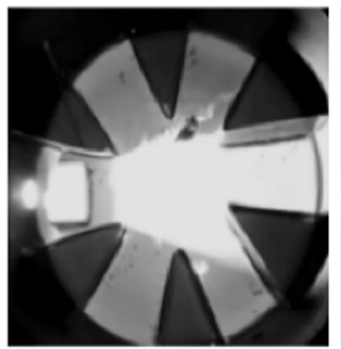

305

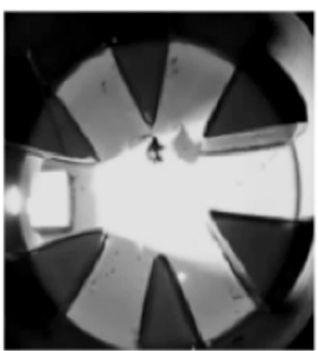

455

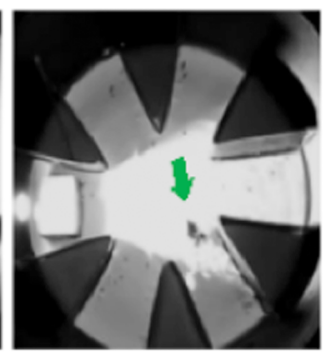

605

(b)

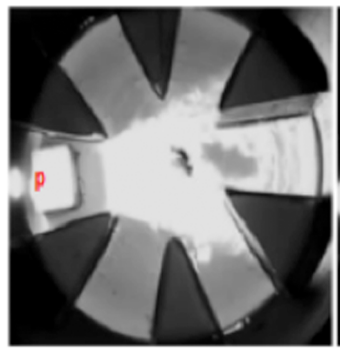

Os

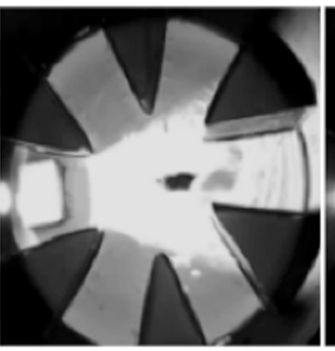

$0.5 \mathrm{~s}$

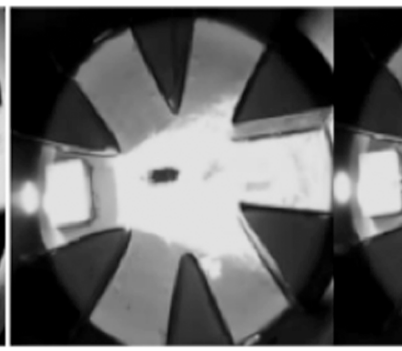

15 (d)

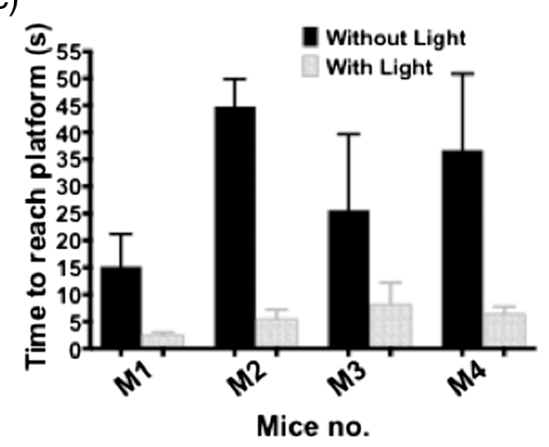

(f)

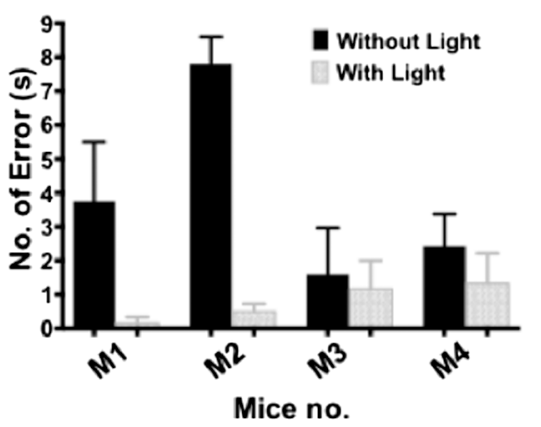

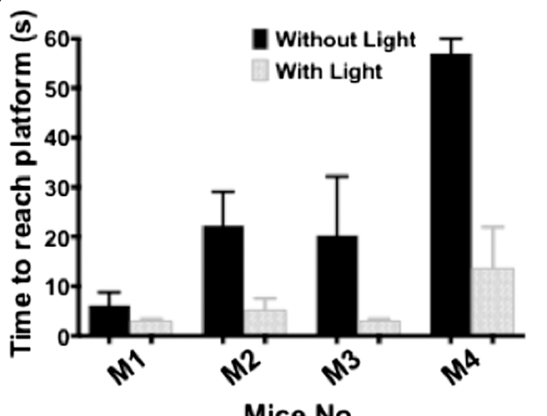

Mice No.

(g)

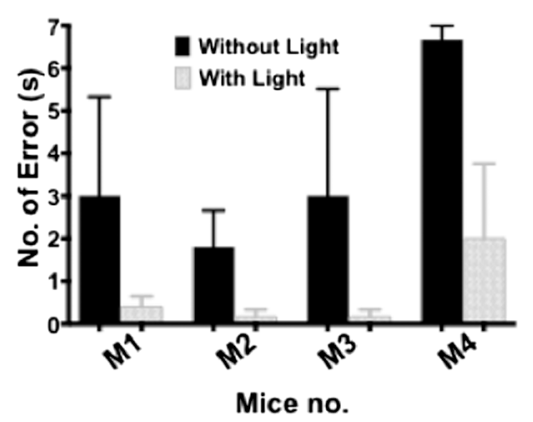

(e)

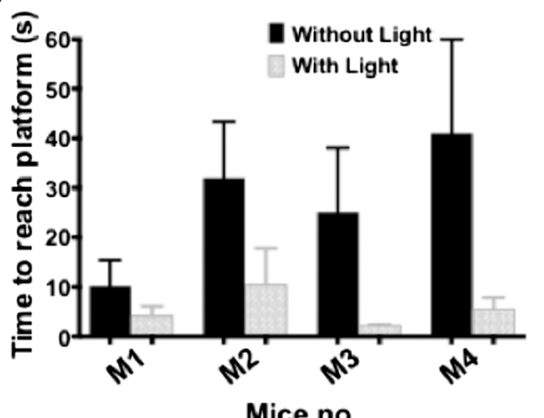

(h)

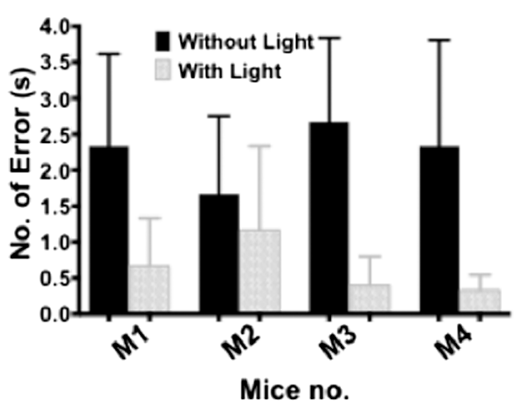

Fig. 4 Time-lapse images of visually guided improvement in rd10 mice behavior in radial water maze. (a) Behavior with white LED light before intravitreal MCO1 injection. (b) Behavior of same mouse at same LED light intensity six weeks after MCO1 injection. Latency to find the platform by the mouse, with and without light, dropped at center (c), side arms 2 and 4 (d), and edge arm 3 (e). Number of error arms traversed by the mouse dropped at different locations before finding the platform in the presence and absence of light: center (f), side arms (g), and edge (h). Average $\pm \mathrm{SEM}, n=5$ for each mouse.

caspase-3 (green)- and DAPI (blue)-stained retina of wild-type mice after chronic light exposure. The number of apoptotic retinal cells was quantified, normalized to the total number of cells, and compared between the MCO1-transfected $r d 10$ and control (wild type) groups. No statistically significant difference was found between these two groups [Fig. 5(d)]. There is no significant cell death in either the wild type or vMCO1-injected $r d 10$ mice, indicating that there is no compromise of cell viability of vMCO1-treated $r d 10$ mice under chronic light exposure. While avoidance of the bright light by the vMCO1-treated mice [Fig. 5(a)] demonstrated light sensitivity of the MCO1-sensitized retina, it is possible that the mice were not fully exposed 

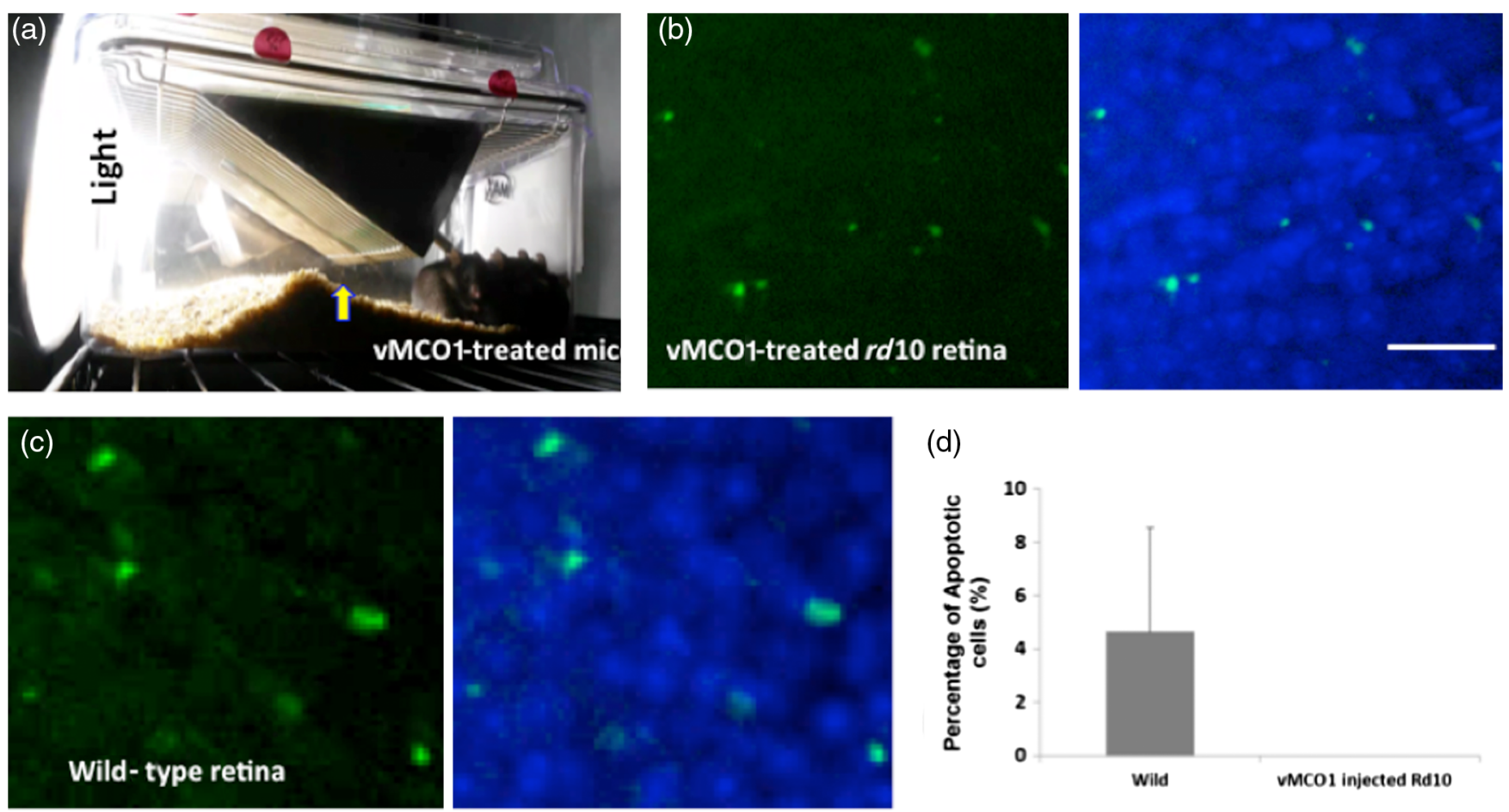

(d)

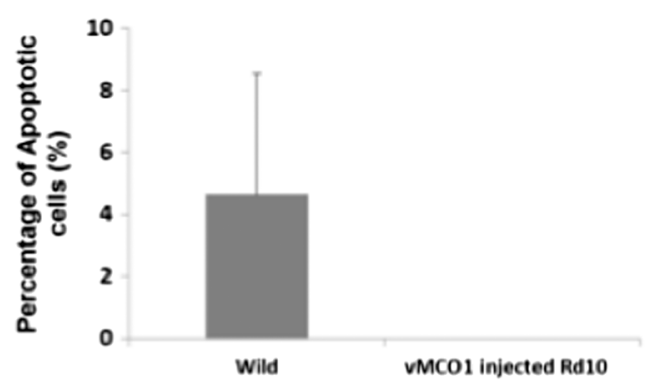

Fig. 5 Viability of retinal cells subsequent to chronic light exposure. (a) Similar to the wild-type (nonblind) mice, vMCO1-treated $r d 10$ mice avoided bright light by staying away and blocking light (via creating a hill out of bedding material, as shown in the arrow). Fluorescence images of retina stained with caspase-3 (green) and overlay with DAPI (right) for vMCO1-treated (b) and wild-type (c) mouse, 4 weeks after $8 \mathrm{~h} /$ day illumination of white light (intensity: $0.1 \mathrm{~mW} / \mathrm{mm}^{2}$ ). (d) Quantitative comparison of \% apoptotic retinal cells between wild-type and vMCO1-treated $r d 10$ mice. Scale bar is $25 \mu \mathrm{m}$. $0 \%$ apoptotic cells in vMCO1.

to chronic illumination all the time during this study $(8 \mathrm{~h} /$ day, 4weeks). Furthermore, since light sensitivity of MCO1-expressing cells significantly reduces the required light intensity for generating $\mathrm{AP}$, the use of vMCO1 minimizes light-induced chronic damage to the retinal cells as compared with those sensitized by ChR2.

\section{Conclusions}

Virally delivered vMCO1 will lead to a clinical approach for treating patients with advanced retinal dystrophies by conventional intravitreal injection of vMCO1. We strongly believe that such treatment will lead to restoration of vision in RP and dry-AMD patients in an ambient light environment in a noninvasive manner without requiring surgery and/or active stimulation methods.

\section{Disclosures}

The authors Sulagna Bhattacharya and Samarendra Mohanty have equity interest in NanoScope Technologies LLC.

\section{Acknowledgments}

The authors would like to thank the National Eye Institute Grants 1R43EY025905-01, 1R43EY026483-01, 3R43EY025905-01S1, and 1R01EY025717-01A1.

\section{References}

1. A. Takeda et al., "CCR3 is a target for age-related macular degeneration diagnosis and therapy," Nature 460(7252), 225-230 (2009).
2. C. A. Curcio, N. E. Medeiros, and C. L. Millican, "Photoreceptor loss in age-related macular degeneration," Invest. Ophthalmol. Visual Sci. 37(7), 1236-1249 (1996).

3. A. Horsager et al., "Predicting visual sensitivity in retinal prosthesis patients," Invest. Ophthalmol. Visual Sci. 50(4), 1483-1491 (2009).

4. C. de Balthasar et al., "Factors affecting perceptual thresholds in epiretinal prostheses," Invest. Ophthalmol. Visual Sci. 49(6), 2303-2314 (2008).

5. E. Zrenner et al., "Subretinal electronic chips allow blind patients to read letters and combine them to words," Proc. Biol. Sci. 278(1711), 1489-1497 (2011).

6. M. Humayun, J. Dorn, and L. da Cruz, "Interim results from the international trial of second sight's visual prosthesis," Ophthalmology 119, 779-788 (2012).

7. G. Nagel et al., "Channelrhodopsin-2, a directly light-gated cationselective membrane channel," Proc. Natl. Acad. Sci. U. S. A. 100(24), 13940-13945 (2003).

8. E. S. Boyden et al., "Millisecond-timescale, genetically targeted optical control of neural activity," Nat. Neurosci. 8(9), 1263-1268 (2005).

9. G. Miller, "Shining new light on neural circuits," Science 314(5806), 1674-1676 (2006).

10. F. Zhang et al., "Circuit-breakers: optical technologies for probing neural signals and systems," Nat. Rev. Neurosci. 8(8), 577-581 (2007).

11. F. Zhang et al., "Channelrhodopsin-2 and optical control of excitable cells," Nat. Methods 3(10), 785-792 (2006).

12. S. K. Mohanty et al., "In-depth activation of channelrhodopsin 2-sensitized excitable cells with high spatial resolution using two-photon excitation with a near-infrared laser microbeam," Biophys. J. 95(8), 3916-3926 (2008).

13. A. D. Bi et al., "Ectopic expression of a microbial-type rhodopsin restores visual responses in mice with photoreceptor degeneration," Neuron 50(1), 23-33 (2006).

14. P. S. Lagali et al., "Light-activated channels targeted to ON bipolar cells restore visual function in retinal degeneration," Nat. Neurosci. 11(6), 667-675 (2008). 
15. M. M. Doroudchi et al., "Virally delivered channelrhodopsin-2 safely and effectively restores visual function in multiple mouse models of blindness," Mol. Ther. 19(7), 1220-1229 (2011).

16. A. Sengupta et al., "Red-shifted channelrhodopsin stimulation restores light responses in blind mice, macaque retina, and human retina," EMBO Mol. Med. 8(11), 1248-1264 (2016).

17. Z. V. Guo, A. C. Hart, and S. Ramanathan, "Optical interrogation of neural circuits in Caenorhabditis elegans," Nat. Methods 6(12), 891-896 (2009).

18. J. Y. Lin et al., "ReaChR: a red-shifted variant of channelrhodopsin enables deep transcranial optogenetic excitation," Nat. Neurosci. 16(10), 1499-1508 (2013).

19. S. Batabyal et al., "Broad-band activatable white-opsin," PLoS One 10(9), e0136958 (2015).

20. K. Dhakal et al., "Optical delivery of multiple opsin-encoding genes leads to targeted expression and white-light activation," Light Sci. Appl. 4(11), e352 (2015).

21. S. Batabyal et al., "Broadband activation by white-opsin lowers intensity threshold for cellular stimulation," Sci. Rep. 5, 17857 (2015).

22. P. Degenaar et al., "Optobionic vision-a new genetically enhanced light on retinal prosthesis," J. Neural Eng. 6(3), 035007 (2009).

23. N. C. Klapoetke et al., "Independent optical excitation of distinct neural populations," Nat. Methods 11(3), 338-346 (2014).

24. H. Hodges, "Maze procedures: the radial-arm and water maze compared," Cognit. Brain Res. 3(3-4), 167-181 (1996).

25. D. Palanker et al., "Design of a high-resolution optoelectronic retinal prosthesis," J. Neural Eng. 2(1), S105-S120 (2005).

Weldon Wright obtained his BS degree in electrical engineering from Southern Methodist University, his MD from the University of Texas Southwestern (UTSW) Medical Center, followed by Ophthalmology Residency at UTSW, fellowship in orbital magnetic resonance imaging at the Jules Stein Eye Institute of the University of California Los Angeles, and a fellowship in Pediatric Ophthalmology at the Wilmer Eye Institute of Johns Hopkins University. He is an experienced clinician with more than 30 years of experience in patient management and clinical research. He has evaluated and treated a broad range of developmental and degenerative diseases, and his expertise includes signal processing and visual electrophysiology.

Sivakumar Gajjeraman has more than 15 years of research experience in advance biomedical science. He is an expert in molecular biology, tissue regeneration, advanced spectroscopic/imaging/x-ray crystallographic, and cell biological techniques. During his previous research tenure at Northwestern and Brown University, he successfully developed methodologies to elucidate the mechanism of various pathological processes at the molecular level using advanced multidisciplinary techniques.

Subrata Batabyal has expertise that span across the area of biophysics, biochemistry, optics, biomedical instrumentation, and optogenetics. He earned his $\mathrm{PhD}$ in chemistry with a focus on ultrafast laser spectroscopy to investigate biomolecular interaction. During his postdoctoral fellowships, he worked on ultrafast laser-assisted gene delivery, optical stimulation, and development of label-free optical techniques to detect neuronal activity and cellular membrane fluctuations.

Sanjay Pradhan carried out postdoctoral research at the University of Alabama, Birmingham, Baylor Institute of Immunology, and UTSW Medical Center. His expertise includes preclinical and clinical models in understanding disease mechanism that includes immune-inflammatory disorders, tumor microenvironment, nerve biology, and skin disease. He has worked on pharmaceutical/medical drug/device development.

Sulagna Bhattacharya has more than a decade of experience in intelligence statistics and analysis. She obtained her degree in electronics engineering. She is an expert in instrumentation, quality control, assurance, software, and animal behavioral analysis.

Vasu Mahapatra graduated from Southlake Carroll High School, Texas, and did his internship at Nanoscope Technologies. He is highly motivated to continue biomedical research. He was awarded the US Air Force Best Science Fair Project Award, Fort Worth Regional Science and Engineering Fair (2016), and was a first place winner in Medical and Health Sciences Division, Fort Worth Regional Science and Engineering Fair (2015).

Ashutosh Tripathy graduated from Lone Star High School, Frisco, Texas, and did his internship at Nanoscope Technologies. He is a member of the Boy Scouts of America, Health Occupational Students of America, member of the Lone Star High School Chapter of National Honors Society, and part of the Frisco Mayor's Youth Council (2015 to 2016).

Samarendra Mohanty has almost two decades of scientific, industrial, and academic experience in biomedical technologies. He is an expert in the design of biomedical optical instrumentation, biophotonics, microscopy, spectroscopy, and therapy. He has authored and coauthored over 200 journal publications and conference presentations. He has cofounded several companies and serves on editorial boards of journals. He was awarded the NEI Audacious Goal Initiative Grant and was a finalist for the NIH Director New Innovator award. 